\title{
Theory on neutrino self-energy and neutrino oscillation with consideration of superconducting energy gap and Fermi's golden rule
}

\author{
Shinichi Ishiguri \\ Nihon University \\ 1-2-1 Izumi-Cho, Narashinoshi, Chiba 275-8575 JAPAN \\ TEL: +81-47-474-9143 \\ Email: ishiguri.shinichi@nihon-u.ac.jp
}

\begin{abstract}
We herein described an investigation of a theory, which describes the energies of neutrinos and the source of neutrino oscillations. A series of experiments were conducted to show evidences of the existence a neutrino mass. We also applied theories to explain the reason for the extremely small energy of a neutrino, mainly by employing a vacuum-derived superconducting energy gap from the Bardeen-Cooper-Schrieffer ground state. Moreover, we succeeded in obtaining the transition probabilities of neutrinos' flavors (i.e., in terms of neutrino oscillation).

We focused on the fact that up- and down-quantized space pairs combine by the Lorentz forces, undertake Bose-Einstein condensation, and then create a superconducting energy gap at the energy level of the vacuum with quantum mechanics fluctuation. Eventually, the superconducting energy gap vanishes to form a real body of the neutrino. Furthermore, assuming that the speed of the neutrino is near the speed of light and exhibits Planck's blackbody emissions, we derived many-body interactions of neutrinos and applied them in Fermi's golden rule. As a result, the neutrino energy we calculated agreed well within the realms of the experimental results. The calculated transition probabilities of neutrino's flavor also explain the experiment results very well.
\end{abstract}

Keywords: neutrino, neutrino's flavor, neutrino oscillation, many-body interactions, superconducting energy gap, a quantized space-time, zero-point energy, lepton, Fermi’s golden rule, mass gap

\section{Introduction}

In our previous articles [1,2], we presented a theory that brings into unison all the fields of particle physics, such as gravitational force, electromagnetic force, weak interaction, strong interaction, and quantization of the Einstein gravity equation under the concepts of quantized time-space and sole 
energy source (which we call therein as the zero-point energy). Essentially, we have defined the meaning of a $\beta$ collapse in these articles, but did not describe the characteristic of neutrinos.

The present article introduces a theory, which describes the self-energy of a neutrino, as well as the neutrino oscillations. This study is significant in such a way that without presenting numerical calculations, it provides an analytical prediction of all the self-energies of neutrino's flavor, which agree well with the current measurements. This study also provides a prediction of neutrino oscillations, such as concrete calculations of the changing probability from an e-neutrino to another neutrino's flavor, which conforms to the measured values. Regarding the proposed theory, it should be noted that any existing theory does not provide a complete and elaborate explanation of why the selfenergy of a neutrino is extremely small and why the neutrino's flavor had such a varying probability that tends to exhibit imbalanced probabilities. Our theory, in essence, elucidates the system of creating the neutrinos, which another theory has yet to perform.

Indeed, this article highlights two points with regard to neutrinos:

1) It answers the question on why neutrinos have extremely small masses and provides concrete calculations to prove it.

2) It presents an elaborate discussion on neutrino oscillations, along with calculations of concrete probability values from e-neutrino to another neutrino's flavor. For this, we describe the neutrino oscillations with regard to a quantized time-space, in particular, the zero-point energy.

Insofar as the standard model is concerned, it predicted that the effective mass of a neutrino is zero because its mass undergoes some form of degeneracy. However, several studies $[3,4,5]$ refuted this statement after discovering neutrino oscillation, which clarified that neutrinos indeed have masses, although they are extremely small. Since then, many theories have tried to explain these very small masses [6,7]. In general, researchers do not consider a neutrino as a Dirac particle, but a Majorana particle. The most well-known theory that could probably explain such mass phenomenon in a neutrino is the seesaw model [6], which predicts that the mass product of a right-winding Majorana neutrino and a left-winding one essentially gives the Dirac particle mass. Such assertion claims that the masses of the right- and left-winding neutrinos depict serious imbalance that a very heavy right-winding neutrino would result to the other neutrino being small. In this theory, however, as it could be assumed that a very heavy right-winding Majorana neutrino must demand the energy of the grand unified theory scale of $10^{15} \mathrm{eV}$, and because the collapse of a proton has not been measured yet, then it is not valid to give an extremely large mass to only the right-winding Majorana neutrino. Unfortunately, there is no existing theory that could predict such extremely small energy of a neutrino. Herein, quantized spacetimes were embedded with up- and down-spin leptons; these electrons combine with each other due 
to magnetic field energy (i.e., gravitational energy) [1], and thus, they behave as a boson. By principle, bosons in the universe follow the Bose-Einstein condensation, which then produces a superconducting energy gap that can be considered as extremely small. Consequentially, if we are to consider the mass gap of leptons, this superconducting energy gap exists at their vacuum energy level as a quantum mechanics fluctuation. Indeed, as defined by the uncertainty relation, the superconducting energy gap vanishes and paves way for the existence of a neutrino real body. In fact, the rest energy for a neutrino cannot be considered (i.e., due to the very high speed, we cannot use the Taylor series to approximate the energy predicted by the relative theory), and the CP symmetry automatically breaks.

Accordingly, no study so far has been associated with the theoretical investigation of neutrino oscillation. Based on the analogy of the Cabibbo-Kobayashi-Maskawa matrix, the Pontecorvo-MakiNakagawa-Sakata metrics [8,9] could be considered, but because of the various problems it is faced with, only the two-generation neutrinos, instead of the three-generation ones, could be taken into account. Nonetheless, even for this case, the mixture angle that expresses the probabilities of transitions between the neutrino's flavors is solely determined through experiments, and thus, a pure theory that can predict such transitions is considered to be currently nonexistent.

As such, in our present study, we claim that:

1. The transition occurs at a quantized time;

2. The thermal equilibrium temperature at a local quantized space-time, at which many neutrinos have interactions with each other, is constant; that is, the solar temperature is not the main cause of neutrino oscillations;

3. The probability of transitions of neutrino's flavors can be calculated using Fermi's golden rule.

The results of our calculations will clarify that each energy gap of neutrino's flavors agrees with the experimental data and that the transition probabilities beginning with an e-neutrino are also consistent with the measured values. We have not considered special undetermined parameters in the calculations in order to demonstrate the validity of the results based on the concepts we have employed.

Our article is structured as follows. Firstly, we provide a brief review about the concepts of a quantized time-space and the zero-point energy [1]. Secondly, we carry out calculations to determine the attractive force binding each quantized time-space. We next discuss the result of applying the Bardeen-Cooper-Schrieffer (BCS) theory in our calculations. Moreover, we introduce Planck's blackbody emission principle in order to consider interactions between the neutrinos. Eventually, we apply the results to Fermi's golden rule. Finally, an Appendix has been presented to describe the solution on the spin problem of a proton. 


\section{Theory}

\subsection{The zero-point energy and concept of a quantized space-time}

The concept of quantized space-time, as well as the zero-point energy, can be especially elaborated in a vacuum condition. As such, we begin by describing each concept by solving the Dirac equation. The equation shows that inside a vacuum, during formation of electron and positrons, a mass gap that exists between them can be represented by the relation

$$
\hbar \omega_{0}=2 m_{e} c^{2},
$$

in which $\omega_{0}, \mathrm{~m}_{\mathrm{e}}$, and c are the angular frequency, electron mass, and speed of light, respectively.

Equation (1) can be interpreted in the form

$$
\frac{1}{2} \hbar \omega_{0}=m_{e} c^{2},
$$

where the term in the left-hand side is identical to the expression of zero-point energy based on the Hamiltonian of the harmonic oscillation

$$
\mathrm{H}=\left(\mathrm{n}+\frac{1}{2}\right) \hbar \omega_{0} .
$$

Note that the first-term in the expanded form of Eq. (3) relates to time-dependent electromagnetic fields, which are calculated in depth in the quantum mechanics, whereas the second term relates to time-independent electromagnetic fields. Indeed, this zero-point energy expression is related to the static electromagnetic fields. If the angular frequency $\omega_{0}$ is constant, then the zero-point energy should be considered as the universal specific constant energy. Equation (3) also describes the energy gap of the vacuum based on the result of the Dirac equation. Therefore, the zero-point energy could be taken as an expression of the basic energy of the vacuum. As we have discussed in [1], the mass of an electron is the most basic parameter; thus, Eq. (2) provides a constant quantized space $\lambda_{0}$ and a quantized time $t_{0}$, which are defined as follows:

$$
\begin{aligned}
& \lambda_{0}=\frac{\hbar}{2 m_{e} c}, \\
& t_{0}=\frac{\hbar}{2 m_{e} c^{2}} .
\end{aligned}
$$

Consequently, we can derive a more general equation of constant quantized time-space length and time as follows:

$$
\begin{aligned}
& \lambda_{c}=\lambda_{0} \sqrt{1-\frac{v^{2}}{c^{2}}}, \\
& t_{c}=t_{0} \sqrt{1-\frac{v^{2}}{c^{2}}} .
\end{aligned}
$$

As will be discussed later, a neutrino does not have rest energy, so that we can consider the above time-space from the lightest Dirac particle, the electron, as the basic quantized time-space. 


\subsection{Principle of creations of neutrinos}

Accordingly, we have described in [1] that the gravitational force or Lorentz force from the magnetic fields comes from the fact that up and down leptons that are embedded in a quantized space-time $\lambda_{c}$ form rotations and then combine with each other as paired quantized space-time because of the attractive force from the Lorentz force or gravity. This paired quantized space-time behaves like a boson. A schematic representation of this phenomenon is presented in Fig. 1. Here, if these bosons experience a Bose-Einstein condensation, a superconductive energy gap is produced on top of the mass gap of the leptons, such as an electron and a positron. This superconducting energy gap gives the real bodies of neutrinos.

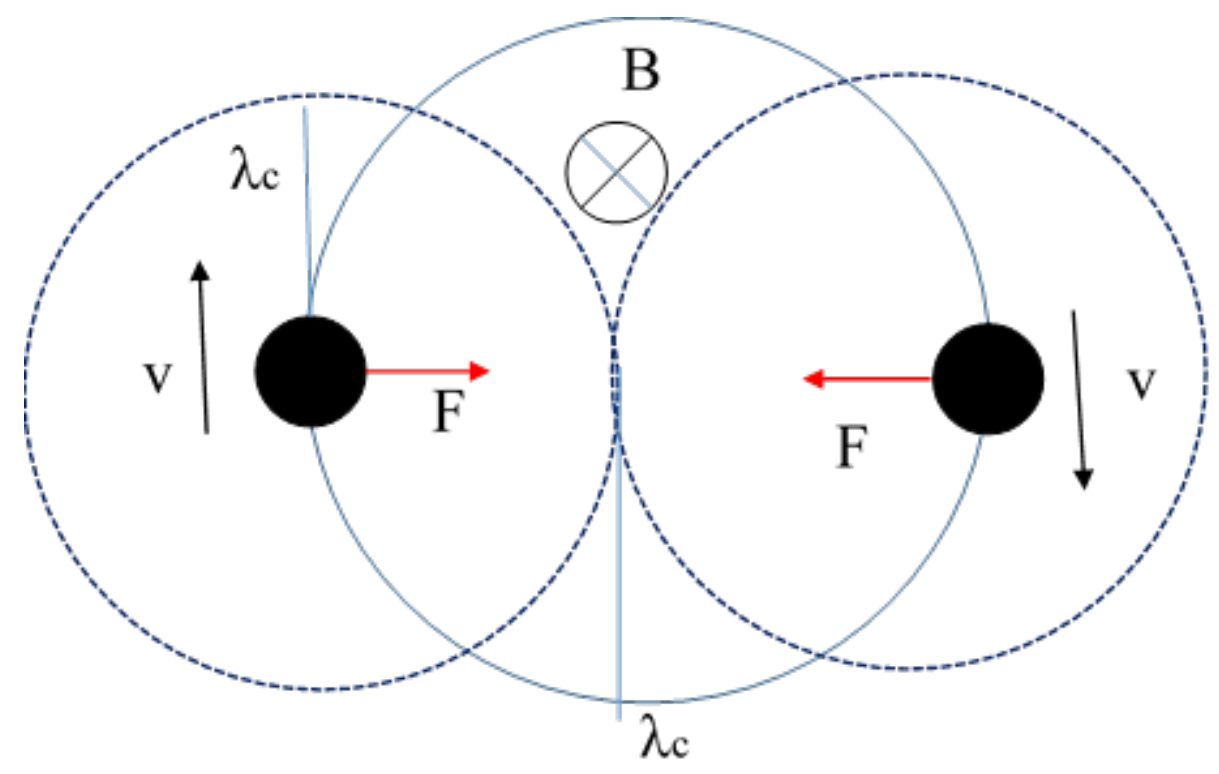

Fig. 1. A schematic model describing a quantized force F. The up- and down-spin electrons do not have real bodies but are embedded in a quantized space. That is, each of the two quantized spaces rotates to create a quantized magnetic field energy, which then produces the Lorentz forces F, which are equal to the attractive gravitational force $\mathrm{F}$.

\section{3 calculation of rotational velocity $v$}

The constant rotational velocity is calculated as follows: 


$$
\begin{aligned}
& \frac{1}{2} \hbar \omega=\frac{1}{2} \hbar \frac{1}{\lambda_{c}} v=m_{e} c^{2} \\
& \mathrm{v}=\frac{2 m_{e} c^{2}}{\hbar} \lambda_{c} \\
& \mathrm{v}=\frac{2 m_{e} c^{2}}{\hbar} \lambda_{0} \sqrt{1-\frac{v^{2}}{c^{2}}} \\
& \mathrm{v}=\frac{1}{\sqrt{2}} c=0.68 c
\end{aligned}
$$

Here, note that a Lorentz contraction is embedded in Eq. (8).

\section{4 calculation of the attractive force between quantized space-time}

Figure 1 describes the combination of quantized space-times at which each lepton (i.e., electron) is embedded, and as mentioned in the previous sections, this paired quantized space-time behaves like a boson, so that we can expect the phenomenon of Bose-Einstein condensation in the entire universe. Moreover, this fact implies that, as a result of the many-body interactions of quantized space-times at which leptons are embedded, a superconducting energy gap that is distinct from the mass gap of electrons and is extremely smaller than the mass gap of electrons in the vacuum is produced. Eventually, as the superconducting energy gap is consumed, each neutrino appears as real bodies.

We investigated this superconducting energy gap by first obtaining concrete calculations of the attractive force $\mathrm{V}_{\mathrm{i}, \mathrm{e}}$ described in Fig. 1, with the purpose of proving that the force is constant in kspace. Following the proof, we can apply the result of the BSC theory immediately.

The electron current derived from the rotation can be defined as

$$
\mathrm{I}=\frac{2 e}{T}
$$

where $\mathrm{T}$ and e indicate the period and the charge of an electron.

The period $\mathrm{T}$ is expressed as

$$
\mathrm{T}=\frac{2 \pi \lambda_{c}}{0.68 c}
$$

Combining the two equations, we can rewrite the current into the form

$$
\mathrm{I}=0.21 \frac{c e}{\lambda_{c}}
$$

Moreover, we can express the central magnetic field derived from the charged electrons' rotations as

$$
\mathrm{B}=\frac{I}{2 \lambda_{c}}=0.1 \frac{c e}{\lambda_{c}^{2}}
$$

As we have mentioned somewhere, the attractive force is equal to the Lorentz force (i.e., the gravitational force in a quantized time-space): 


$$
\mathrm{F}=\mathrm{evB}=0.068 c^{2} \frac{e^{2}}{\lambda_{c}^{2}}
$$

where a quantized space $\lambda_{c}$ can be approximated as

$$
\lambda_{c} \approx \lambda_{0} \text {. }
$$

As such, we can use integration to find the attractive potential $\mathrm{V}_{\mathrm{i}, \mathrm{e}}$,

$$
V_{l, e}=-\int F d r=-0.068 c^{2} \frac{e^{2}}{\lambda_{c}} \approx 0.068 c^{2} \frac{e^{2}}{\lambda_{0}}=-8.0 \times 10^{-10} \mathrm{~J},
$$

which clarifies that $\mathrm{V}_{\mathrm{i}, \mathrm{e}}$ is constant along the velocity space (i.e., $\mathrm{k}$-space).

On this regard, we are allowed to employ the BCS theory.

Moreover, since $\lambda_{0}$ is inversely proportional to the mass of the electron, other forces, such as $V_{\mathrm{i}, \tau}$, can be derived from the ratio of the mass of leptons over that of the electron, i.e., the product by the parameter $\eta$ (which indicates the ratio of $\mu$ - or $\tau$-lepton mass for the mass of e- lepton) creates the other forces.

\section{5 derivation of a superconducting energy gap in the vacuum}

Accordingly, the BCS theory states that the superconducting energy gap can be derived using the formula

$$
2 \Delta_{v, e}=\hbar \omega_{D} \exp \left(-\frac{1}{V_{l, e} N}\right)
$$

where $\mathrm{N}$ is the density of states.

Nonetheless, note that state density in Eq. (19) is extremely large if we are to consider the entire universe. On this regard, we can use approximate Eq. (19) into

$$
2 \Delta_{v, e}=\hbar \omega_{D}=k_{B} T,
$$

in which $\mathrm{k}_{\mathrm{B}}$ and $\mathrm{T}$ represent the Boltzmann constant and a constant temperature, respectively.

However, $\mathrm{T}$ can be changed based on two reasons:

(i) According to the state equation of gases,

$$
\mathrm{T}=\frac{V}{2 R} p,
$$

with $\mathrm{V}, \mathrm{R}$, and $\mathrm{p}$ indicating the volume, the universal gas constant, and the pressure of the gas, respectively.

The pressure $\mathrm{p}$, which is proportional to the temperature $\mathrm{T}$, implies the force $\mathrm{V}_{\mathrm{l}, \mathrm{e}}$, and $\mathrm{V}_{\mathrm{i}, \mathrm{e}}$ depends on a quantized space $\lambda_{c}$ (see. Eq. (4). Note that, in this paper, this quantized space $\lambda_{c}$ is approximated as $\lambda_{0}$ ). Therefore, $\mathrm{V}_{\mathrm{l}, \mathrm{e}}$ is proportional to the mass $\mathrm{m}$ of each lepton.

(ii) As a result of the many-body interactions among leptons, the average kinetic energy is expressed as 


$$
\langle e\rangle=\left\langle\frac{1}{2} m v^{2}\right\rangle=\frac{3}{2} k_{B} T .
$$

As such, $\mathrm{T}$ is proportional to mass of each lepton $\mathrm{m}$.

Considering the above, we can obtain a more general expression of the superconducting energy gap:

$$
2 \Delta_{v}=\eta^{2} k_{B} T_{0} .
$$

where $\eta$ denotes a ratio of $\mu$ - or $\tau$-lepton mass for the mass of e-lepton.

We will claim later that neutrino oscillations occur at each quantized time, in an almost the same manner as how an electromagnetic wave (photons) in the vacuum is transmitted as the speed c, which is valid in the relativity theory. On a more concise note, this means that the speed of neutrino is almost the same as the speed of light. Indeed, our calculations in [1] estimated the neutrino speed at 0.993c.

\subsection{Introduction of the many-body parameter $\alpha_{T}$}

The energy of each lepton can be obtained using the equation

$$
E_{l}=\frac{p_{l}^{2}}{2 m}+V_{l},
$$

where $\mathrm{p}_{\mathrm{i}}$ indicates the momentum.

By considering the average $\left\langle\mathrm{E}_{l}>\right.$, the kinetic energies of the leptons are cancelled by each other's interactions, and thus, the many-body interactions create a physical parameter $\alpha_{T}$ that is entirely dependent on the temperature:

$\left\langle E_{i}\right\rangle=\alpha_{T} V_{l}$. (23)

Accordingly, $\alpha_{\mathrm{T}}$ is related to neutrinos. Considering that the mass of a neutrino is extremely small and its speed is near the speed of light, we can assume that $\alpha_{\mathrm{T}}$ functions similarly to Planck's blackbody emissions:

$$
\alpha_{T} \propto \frac{\hbar \omega}{\pi^{2} c^{2}} \frac{1}{\exp \left(\frac{\hbar \omega}{k_{B} T_{0}}\right)-1} .
$$

It should be noted that $\alpha_{T}$ is a dimensionless parameter; thus, Planck's constant and the volume dimensions can be removed:

$$
\alpha_{T} \equiv \frac{1}{\pi^{2}} \frac{1}{\exp \left(\frac{\hbar \omega}{k_{B} T_{0}}\right)-1}=\frac{1}{\pi^{2}} \frac{1}{\exp \left(\frac{\hbar c}{\lambda_{T^{k} T_{0} T_{0}}}\right)-1} .
$$

According to speckles of Planck's blackbody emission [10], the wavelength $\lambda_{\mathrm{T}}$ is distributed in the order of $10^{-6} \mathrm{~m}$, but here, we assume that the temperature is room temperature $300 \mathrm{~K}$. As such, $\lambda_{\mathrm{T}}$ can be approximated as

$$
\lambda_{T} \approx 6.5 \times 10^{-6} \mathrm{~m}
$$


The same distributions of e-, $\mu$-, and $\tau$-neutrinos are found in a quantized space, wherein they behave as Planck's blackbody emissions. This means that even after considering solar neutrinos, the temperature of the sun is not in a way very related to the probabilities of transitions between the neutrino's flavors but rather to the temperature of each quantized time-space in the vicinity $\mathrm{H}_{v}$ of the earth. Here, we can approximate this as the room temperature $300 \mathrm{~K}$ :

$$
H_{v}=\left|\left\langle E_{e}\right\rangle-\left\langle E_{\mu, \tau}\right\rangle\right|=\alpha_{T}\left(V_{l, \mu: \tau}-V_{l, e}\right) .
$$

Equation (27) shows that $\mathrm{H}_{v}$ depends on the temperature difference but that it works at many-body interactions of neutrinos (not leptons) in a quantized time-space. As a result of this neutrino oscillation, each temperature in a quantized space-time achieves the local temperature of the thermal equilibrium.

\section{7 wave functions of neutrinos and the Fermi's golden rule}

For e-, $\mu$-, and $\tau$-neutrinos, their wave functions are defined as follows:

$$
\begin{aligned}
& \psi_{v, \tau}=\left|\psi_{v}\right| \exp \left(i \omega_{D, \tau} t\right), \\
& \psi_{v, \mu}=\left|\psi_{v}\right| \exp \left(i \omega_{D, \mu} t\right), \\
& \psi_{v, e}=\left|\psi_{v}\right| \exp \left(i \omega_{D, e} t\right) .
\end{aligned}
$$

for which the normalization

$$
\int\left|\psi_{v}\right|^{2} d v \equiv 1
$$

holds.

More importantly, each neutrino wave function $\lceil\psi\rceil$ is common in all expressions, and as mentioned in the previous sections, the momentum factor is not included in these wave functions since neutrino interactions (i.e., neutrino oscillations) presumably occur at each quantized time-space. Here, the key point is that each neutrino wavelength is approximately $\lambda_{T}=6.5 \times 10^{-6} \mathrm{~m}$, which is exceedingly larger than that of a quantized time-space, and thus, the superposition of Eqs. (28-1), (28-2), and (283) can be formed. For the same reason, it is accurate to say that the distribution $|\psi|^{2}$ is the same within a quantized time-space; that is, there are no points for a neutrino in a quantized time-space. Moreover, in each experiment, basic line $\mathrm{D}[\mathrm{km}]$ from the source to the location of measurement is one of the parameters considered: the larger the $\mathrm{D}$, the larger the many-body interactions of the neutrinos, which prompt us to measure sharper neutrino oscillations. However, it does not necessarily imply that the probability of transitions increases. On such note, we recommend that neutrino oscillations be measured from the solar emissions because the width of the earth's atmosphere is sufficiently large.

As a result of the interactions of the neutrinos with each other, the probability of transitions for neutrino's flavors can be calculated by using Fermi's golden rule.

For example, the probabilities from e-neutrino include

$$
\mathrm{P}(\tau \rightarrow e)=\left\langle\psi_{v, \tau}\left|\alpha_{T}\left(V_{l, \tau}-V_{l, e}\right)\right| \psi_{v, e}\right\rangle^{2} \frac{2 \pi}{\hbar} t_{c} \delta\left(\omega_{D, \tau}-\omega_{D, e}\right),
$$




$$
\mathrm{P}(\mu \rightarrow e)=\left\langle\psi_{v, \mu}\left|\alpha_{T}\left(V_{l, \mu}-V_{l, e}\right)\right| \psi_{v, e}\right\rangle \frac{2 \pi}{\hbar} t_{c} \delta\left(\omega_{D, \mu}-\omega_{D, e}\right)
$$

In both the above equations, $\mathrm{t}_{\mathrm{c}}$ is a quantized time; here, we consider the approximation

$$
t_{c} \approx t_{0}=\frac{\hbar}{2 m_{e} c^{2}}
$$

where $m_{e}$ represents the mass of an electron. We can employ quantized time, which stems based on the fact that the speed of neutrino is very close to the speed of light c.

\section{8 lifetime of a superconducting energy gap}

The superconducting energy gap we described here does not survive permanently. Due to the uncertainty relation, the lifetime of a superconducting energy gap is calculated.

In particular, the superconducting energy gap is expressed by the relation

$$
2 \Delta_{v}=k_{B}\left(\eta^{2} T_{0}\right)=2 \Delta E \text {. }
$$

By employing the uncertainty relation,

$$
\frac{1}{2} k_{B}\left(\eta^{2} T_{0}\right) \Delta t_{v} \approx \hbar,
$$

where $\Delta t_{v}$ indicates the lifetime of a superconducting energy gap and is expressed as

$$
\Delta t_{v}=\frac{2 \hbar}{k_{B}\left(\eta^{2} T_{0}\right)} .
$$

As an example, if we assume the temperature to be room temperature $300 \mathrm{~K}$, then the lifetime of a superconducting energy gap for an e-neutrino is

$$
\Delta t_{v, e}=5.3 \times 10^{-14} \mathrm{~s} .
$$

Similarly, the lifetime of the superconducting energy gap for a $\tau$-neutrino is

$$
\Delta t_{v, \tau}=3.0 \times 10^{-21} \mathrm{~s} .
$$

Indeed, the superconducting energy gaps vanishes spontaneously, giving rise to the real bodies of neutrinos.

\section{Results and Discussion}

In table1, we show the physical constants using calculations. 
Table 1 The physical constants for calculations in this study

Local equilibrium temperature $T_{0}$

$300 \mathrm{~K}$

Mass ratios of lepton for an electron $\eta$

$\begin{array}{lc}\text { to Electron lepton } & 1 \\ \text { to } \mu \text {-lepton } & 207 \\ \text { to } \tau \text {-lepton } & 3484\end{array}$

Boltzmann constant $\mathrm{k}_{\mathrm{B}}$

$1.38 \times 10^{-23} \mathrm{~J} / \mathrm{K}$

Plank constant $\hbar$

$1.05 \times 10^{-34} \mathrm{Js}$

Speed of light c

$3.0 \times 10^{8} \mathrm{~m} / \mathrm{s}$

Wave length of blackbody's emission $\quad \lambda_{\mathrm{T}}$

$6.5 \times 10^{-6} \mathrm{~m}$

Attractive forces between leptons for electrons $\mathrm{V}_{\mathrm{l}, \mathrm{e}}$

$8.0 \times 10^{-10} \mathrm{~J}$

for $\mu$-leptons $\mathrm{V}_{\mathrm{i}, \mu}$

$\eta \times 8.0 \times 10^{-10} \mathrm{~J}$

for $\tau$-leptons $\mathrm{V}_{\mathrm{l}, \tau}$

$\eta \times 8.0 \times 10^{-10} \mathrm{~J}$

Electron mass $m_{e}$

$9.1 \times 10^{-31} \mathrm{Kg}$

Based on Table 1, we first obtained the neutrino self-energy for each generation as follows:

$$
\begin{gathered}
2 \Delta_{v, \tau}=0.314 \mathrm{MeV}, \\
2 \Delta_{v, \mu}=1.18 \mathrm{KeV}, \\
2 \Delta_{v, e}=0.025 \mathrm{eV},
\end{gathered}
$$

Next, we determined the probabilities of transitions between neutrinos' flavors:

$$
\begin{aligned}
& \mathrm{P}(\mathrm{e} \rightarrow \tau)=0.732, \\
& \mathrm{P}(\mathrm{e} \rightarrow \mu)=0.0021, \\
& \mathrm{P}(\mathrm{e} \rightarrow \mathrm{e})=1-\mathrm{P}(\mathrm{e} \rightarrow \tau)-\mathrm{P}(\mathrm{e} \rightarrow \mu)=0.266 .
\end{aligned}
$$

Now, let us consider the superconducting gaps for each neutrino. In our experiments, each energy was within the inequality boundary [11], and our calculations were in the realms. This validates our claim that each neutrino appears after the superconducting energy gap in each quantized time-space vanishes and that these superconducting energy gaps are produced by many-body interactions with each paired quantized time-space at which a lepton (i.e., an electron) is embedded. Moreover, previous studies have considered the neutrino as a Dirac particle based on the seesaw model, but here, we claimed that the superconducting energy gap in these particles was much smaller than the mass gap of an electron and that it exists as a quantum mechanics fluctuation at the energy level of the vacuum. Therefore, the 
CP symmetry is automatically broken, because these superconducting energy gaps do not necessarily imply mass gaps (i.e., rest energy). This fact can be understood by considering that a normal and cooled superconductor has an energy gap as a result of the many-body interactions of electrons and Cooper pairs and that breaking such superconducting energy gap generally results in the formation of a photon. Thus, we could claim that the source of breaking the CP symmetry differs from the case of the quarks by the Cabibbo-Kobayashi-Maskawa matrix.

Moreover, we claimed that interaction with each neutrino within a quantized time-space results in neutrino oscillation. As such, including that interaction, Fermi's golden rule gave representative transition probabilities. Note that we assumed that the initial neutrino from a source is an e-neutrino and calculated the transition for that e-neutrino. Our calculations revealed an important point, that is, the transition from e-neutrino to $\mu$-neutrino is a very rare case, whereas the transition to $\tau$-neutrino is long, in addition to the survival probability of an e-neutrino. Experiment [5] supported these results, including the concrete transition probability values.

In our previous articles [1,2], we have discussed a unified theory, which explains all the particle fields, including the electromagnetic fields, gravitational fields, weak interaction, and strong interaction. This theory employs the sole energy source as the zero-point energy derived from the mass gap of an electron and quantized time-space. In other words, no other serious undetermined parameters were defined, that is, we have considered Planck's constant, the speed of light, and the mass of an electron, which are the components of the zero-point energy and quantized time-space, to explain all the fields. In the process of a $\beta$ collapse, we clarified the reason for the existence of an e-neutrino. The quantized time-space $\lambda_{0}$ in Eq. (4) is of the order of $10^{-13} \mathrm{~m}$, whereas a nucleon is of the order of $10^{-15} \mathrm{~m}$. Thus, in order for an electron to exist in a nucleon without bypassing the concept of a quantized time-space, an e-neutrino orbits around the electron, and thus, a normal quantized time-space (i.e., $10^{-13} \mathrm{~m}$ order) changes to the order of the radius of a nucleon (i.e., $10^{-15} \mathrm{~m}$ order). With this fact, we calculated the mass of a neutrino, which we found out to involve gravitational interaction, and thus, it was not pure self-energy. Hence, we needed to explain what makes the neutrino energy very small and what triggers the neutrino oscillations. According to our results, the up- and down-spin paired quantized time-spaces exhibit many-body interactions among each other, thereby resulting to Bose-Einstein condensation and then the generation of superconducting energy gap. Note that this gap is extremely smaller than the zero-point energy for an electron but is not the Dirac gap, which leads to the automatic breakage of the CP symmetry. More appropriately, this suggests that the superconducting energy gap exists at the energy level in the vacuum with the quantum mechanics fluctuation. Moreover, because of the lifetime of a superconducting energy gap, the gap energy is consumed to pave way for the existence of a real neutrino body. Considering these results, our present study does not pose any contradictions 
to the unified theory in our previous articles.

\section{Conclusion}

This article presented a theoretical description of the reason for the extremely small energy of a neutrino and demonstrated mathematical formulations to calculate the probabilities of the transitions of the neutrino's flavors.

Our experimental investigations clarified the neutrino oscillations; nonetheless, there is no theory yet so far to explain the neutrino energy and neutrino oscillation in the case of a three-generation neutrino. We intend to explore this position in the future.

Furthermore, we showed that the up- and down-quantized time-space pairs having many-body interactions resulted to the Bose-Einstein condensation and the superconducting energy gap. This gap exists at the energy level of the vacuum with quantum mechanics fluctuation, which creates a real body of the neutrino. We also investigated the transitions of neutrinos' flavors by taking into account Planck’s blackbody emissions, formulating many-body interactions by the neutrinos, and employing these interactions in Fermi's golden rule to obtain the transition probabilities.

Our calculated results fell within the neutrino energies suggested by the experimental values. The calculated transition probabilities were also successful in explaining the imbalances in the numbers for each neutrino.

\section{Additional information}

This paper is not related to any competing interests such as funding, employment and personal financial interesting. Moreover, this paper is not related to non-financial competing interesting

\section{Acknowledge}

We thank Enago (www.enago.jp) for English language Review.

\section{References}

[1]S. Ishiguri, A Unified Theory of All the Fields in Elementary Particle Physics Derived Solely from the Zero-Point Energy In Quantized Spacetime, Preprints 2019, 2019070326 (doi:10.20944/prepronts201907.0326.v1) (2019)

[2]S. Ishiguri, Studies on Quark Confinement in a Proton on the Basis of Interaction Potential, Preprints, 2019, 2019020021 (doi: 10.20944/preprints201902.0021.v1)

[3]Y. Fukuda et al [Super-Kamiokande Collaboration], Phys. Rev. Lett. 81, 1562 (1998)

[4]E. Aliu et al [K2K Collaboration], Phys. Rev. Lett. 94, 081802 (2005) 
[5]Q. R. Ahmad et al [SNO Collaboration], Phys. Rev. Lett. 89, 011301 (2002)

[6] T. Yanagida, Prog. Theor. Phys. 64, 1103 (1980)

[7]T. Nakaya, Neutrino Physics (Particle Physics and Cosmology with Neutrinos) pp.12-pp.15 , Kyourits-Shuppan, Tokyo, (2016)

[8]Z. Maki, M. Nakagawa and S. Sakata, Prog. Theor. Phys. 28, 870 (1962)

[9]B. Pontecorvo, Sov. Phys. JETP 7, 172 (1958)

[10]H. Ezawa, Quantum Mechanics, 1, Shokabo, Tokyo, pp.13-pp.17, (2015)

[11] P.A.R. Ade et al [Planck Collaboration], Astron. Astrophys. 571, A16 (2014)

\section{APPENDIX}

\section{A1. Introduction}

So far, it is considered that the summation of spins of quarks results in the spin of a proton. However, according to [a1], the spins of quarks' contributions reach only $30 \%$ of the total spins of a proton. Also, in our article [a2], although the value of spin of each quark is $\pm \frac{1}{6} \hbar$, the summation is $\frac{1}{6} \hbar$, which is $32 \%$ of the spin of a proton $\frac{1}{2} \hbar$. We considered this spin problem in this article. To conclude, the spin of a proton is the total angular momentums $\mathrm{j}$, whose elements could be taken as a summation of spins of quarks and angular momentum $l$. This angular momentum $l$ is naturally introduced by the fact that each quark has rotations to generate magnetic field energy. For details, see our previous article [a2].

\section{A2. Theory}

Let us consider the quantization of a momentum. From both small momentum $\mathrm{p}$ and time $\mathrm{t}$, the following equation holds:

$$
\mathrm{p}=F_{0} t
$$

where $\mathrm{F}_{0}$ is a constant force.

Because time is divided by a quantized time $t_{c}$,

$$
\mathrm{t}=\mathrm{n} t_{c}
$$

where $t_{c}$ in the quark space is derived from the zero-point energy for quark [ a2,a3 ].

In the quark space, the zero-point energy is defined as

$$
\frac{1}{2} \hbar \omega=3 m_{A} c^{2}
$$


in which $\mathrm{m}_{\mathrm{A}}$ indicates the mass of a quark. Note that the first generation of a quark mass is three times that of an electron [a2],

$$
t_{c}=\frac{2 \pi \hbar}{6 m_{A} c^{2}}
$$

Now, let us consider the angular momentums.

With a quantized time-space, we tend to consider that

$$
\mathrm{l}=\mathrm{rp}=\mathrm{n} \lambda_{c} \times n p_{c}=n^{2} l_{0}, \text { (a5) }
$$

where $\mathrm{p}_{\mathrm{c}}$ and $\mathrm{l}_{0}$ are the minimum momentum and minimum angular momentum, respectively.

However, here, we consider a nucleon. As such, due to the existence of e-neutrino, the radius length is not quantized:

$$
\mathrm{n} \lambda_{c} \rightarrow a
$$

where a denotes the radius of a proton.

Moreover, the minimum angular momentum is derived from the uncertainty relation:

$$
\begin{aligned}
& l_{0}=a p_{c} \approx \hbar, \\
& \mathrm{l}=\mathrm{n \hbar} .
\end{aligned}
$$

Considering Eq. (a3),

$$
\omega=\frac{6 m_{A} c^{2}}{\hbar} .
$$

Consequently, the time period $\mathrm{T}$ can be calculated using the expression

$$
\mathrm{T}=\frac{2 \pi \hbar}{6 m_{A} c^{2}}
$$

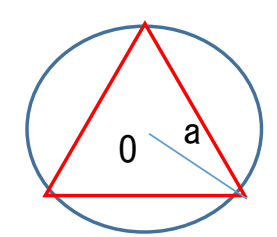

Fig. a1 a model of a proton. Three quarks are located at each apex of equilateral triangle. Maintaining the shape of the triangle, the rotational motion along the origin $\mathrm{O}$ is introduced

As shown in our quark model in Fig. a1, the triangle whose apex denotes each quark having a rotation with the period T, we can use Eq. (a9) to obtain

$$
2 \pi \mathrm{a}=\mathrm{Tv},
$$

where $\mathrm{v}$ is the velocity of the quarks' rotation.

Here, the angular momentum is 


$$
\mathrm{l}=3 m_{A} \times 3 v \times a=9 a m_{A} v,(\mathrm{a} 11)
$$

where the number of quarks is 3 ; thus,

$$
2 \pi \mathrm{a}=\mathrm{T} \frac{l}{9 a m_{A}} .
$$

If we temporally assume the equation

$$
\mathrm{l}=n^{2} \hbar
$$

then the square of integer $n$ becomes

$$
n^{2}=54\left(\frac{m_{A} c}{\hbar}\right)^{2} .
$$

As mentioned, the radius length is not quantized with the compensation of the existence of an eneutrino. Thus, Eq. (a13) can be replaced by

$$
\mathrm{l}=\mathrm{nh} \text {, }
$$

with

$$
\mathrm{l}=\sqrt{54} \frac{m_{A} a c}{\hbar} \hbar \approx \approx 0.336 \hbar \text {. }
$$

Here, the value of a is $0.8 \times 10^{-15} \mathrm{~m}$ (i.e., radius of a proton), and $\mathrm{m}_{\mathrm{A}}$ is three times the mass of an electron.

In our previous article in Appendix [1], we showed that the spin of each quark is calculated as

$$
s= \pm \frac{1}{6} \hbar \text {. }
$$

Thus, the total spin in a proton is

$$
s_{\text {total }}=\frac{1}{6} \hbar,
$$

and the total angular momentum $\mathrm{j}$ is

$$
\mathrm{j}=s_{\text {total }}+l=\frac{1}{6} \hbar+0.336 \hbar=0.496 \hbar \approx \frac{1}{2} \hbar . \text { (a19) }
$$

If we are to measure the spin of a proton as the total angular momentum as above, our result will agree well with the measurements [1a].

\section{Reference}

[a1] Y. Goto, Progress in Nucleon Spin Physics, 72th Japan Physical Society conference, 244 (2017) [a2] S. Ishiguri, A Unified Theory of All the Fields in Elementary Particle Physics Derived Solely from the Zero-Point Energy In Quantized Spacetime, Preprints 2019, 2019070326 (doi:10.20944/prepronts201907.0326.v1) (2019)

[a3] S. Ishiguri, Studies on Quark Confinement in a Proton on the Basis of Interaction Potential, Preprints, 2019, 2019020021 (doi: 10.20944/preprints201902.0021.v1) 